\title{
Comparison of Calibration Methods with and without Feature Selection for the Analysis of HPLC Data
}

\author{
Piedad Parrilla Vázquez, María Martínez Galera, Antonia Garrido Frenich, \\ and José L. Martínez VIDAL ${ }^{\dagger}$ \\ Department of Hydrogeology and Analytical Chemistry, University of Almeria, 04071 Almeria, Spain
}

\begin{abstract}
A comparison of two multivariate calibration methods, partial least squares (PLS) and principal component regression (PCR), applied to high-performance liquid chromatography (HPLC) data, is presented for the resolution of a pesticide mixture. The data set showed both coeluted peaks and overlapped absorption spectra. Besides, there is an additional overlapping between the signal of the mobile phase and that of some pesticide. Multivariate calibration models were evaluated using different criteria to choose the optimum number of latent variables. It is shown that PLS yields the best predictive models. Furthermore, two methods for selecting regions were applied with the goal to achieve an improved prediction ability in the present multicomponent determination by HPLC-DAD (diode array detector) with PLS. The selection of regions associated with a large correlation to the concentration and with large values in loading-weighs (from PLS) were considered. It is concluded that feature selection can also improve the multivariate calibration results using chromatographic data.
\end{abstract}

(Received March 8, 1999; Accepted September 13, 1999)

Well-established multivariate calibration methods, such as principal component regression (PCR) and partial least squares (PLS), have been successfully applied to spectroscopic analyses. ${ }^{1-4}$ Early applications used complete spectra for the determination of all components in a mixture. Nevertheless, increasing evidence, from simulations and from experiments, has showed that these full-spectrum algorithms could also benefit from variable selection, particularly for complex data sets. Several researchers have demonstrated that employing selected wavelengths, single or bands of wavelengths, rather than the entire spectrum, improves the accuracy of predictions..$^{5-29}$

All the same, most of the work in feature selection has been performed in the area of spectroscopic analyses; the elimination of some of them may give simpler models and better precision of the predictions. However, why not apply variable selection in other analytical techniques, such as chromatographic techniques if the quality of multivariate calibration models depends, among others, on the quality of variables? Obviously, it should be noted that the goal here is not a variable selection to find the smallest subset of discrete variables for fitting or predicting a model, but to determine if variable elimination can improve the predictive ability of each component.

The selection of how many and which variables should be retained in the analytical process is usually the most difficult task. Recently, optimisation algorithms, such as simulated annealing and genetic algorithms, have been applied to the selection of individual variables. ${ }^{11,12,17-21,26-28}$ These optimisation methods can take up a long computer time and generally select individual variables. Here, we demonstrate the adequacy of applying simple methods of variable elimination in simultaneous multicomponent chromatographic analyses, and

†To whom correspondence should be addressed.

E-mail: jlmartin@ualm.es we determine their utility for using in conjunction with PLS.

The HPLC-DAD data set used was a mixture of the cyromazine, oxamyl and methomyl pesticides that show both coeluted peaks and overlapped absorption spectra. In these samples there is also an additional overlapping due to the signal of the mobile phase. These pesticides are commonly analysed by HPLC, ${ }^{30-33}$ in despite of it is difficult their separation because of their high polarity.

Reversed-phase liquid chromatography (RPLC) is a more suitable technique for the determination of polar analytes than GC-based techniques, since derivatisation is usually superfluous and in the analysis of aqueous samples the mobile-phase system is fully compatible, resulting in a high potential for automation. Despite everything, in LC it is difficult to efficiently separate polar analytes in one run, because the chromatographer must provide sufficient selectivity between the analytes and between the analytes and the solvent peak at the same time. Given that the polarity of these pesticides is very similar, chemical changes in the mobile phase are not enough to achieve sufficient selectivity. Thus, multivariate calibration is an efficient choice to handle this problem without any previous chemicalseparation step.

In this work a preliminary study was carried out to determine which full spectrum method, PLS or PCR, better handles the overlapping between the compounds and the solvent peak. Also, two different criteria were evaluated to select the optimum number of latent variables (LVs). Next, simple variable elimination methods were applied in combination with PLS. These methods are based on chromatographic or mathematical criteria. One of them is based on a correlation plot, and the other on a loading weigh plot for each component. Evaluation of the optimal regions of variables is based on the minimisation of prediction errors for the calibration set.

Finally, in order to demonstrate the analytical usefulness of the variable elimination, without any loss of prediction capacity, 
it was applied to the determination of these pesticides in groundwater at ppb levels after liquid-liquid extraction (LLE) with dichloromethane.

\section{Description of the Used Method}

In this section a brief description of the full-spectrum methods concerning aspects related to the goal of this work, as well as, the methods for data reduction, are considered.

\section{Full-spectrum multivariate calibration methods}

Let us assume that we have collected a set of calibration data $(X, Y)$, where the matrix of independent variables $(\boldsymbol{X})$ contains the chromatographic data, i.e. the variables measured for each sample, and the dependent variables data matrix $(\boldsymbol{Y})$ contains the variables that depend on the $X$ data, i.e. the concentrations know of the analytes for each sample. Theory indicates that a linear relation is to be expected, e.g. Lambert-Beer's law of the linear relationship between concentration and absorbance. This can be expressed as

\section{$Y=X B+F$}

where $\boldsymbol{B}$ is the matrix of regression coefficients calculated by a statistical analysis and $\boldsymbol{F}$ is the matrix of residuals, not explained by the model.

PLS and PCR ${ }^{1,34,35}$ are both factor or LVs methods that transform the original matrix into the product of two smaller matrices, $\boldsymbol{T}$ and $\boldsymbol{P}$,

$$
X=T P^{\prime}+E
$$

where $\boldsymbol{T}$ is the scores matrix, $\boldsymbol{P}$ is the loading matrix and $\boldsymbol{E}$ is the error matrix. The superscript' indicates a transposed matrix. The combination of both matrices represents the part of the data that makes sense. The error or residual part is the fraction of variation that cannot be interpreted by the model.

This representation of $\boldsymbol{X}$ as TP is called factor analysis, where the absorbances at all times used in the analysis are compressed to a small number of absorbances in a new coordinate system. This new coordinate system is composed of loading vectors that are linear combinations of the original chromatographic calibration data. The amounts of each loading vector which are required to reconstruct each calibration chromatogram are the scores. Thus, PLS and PCR are methods that are concerned with modeling both chromatograms and concentrations during calibration.

PCR is a two-step procedure, which first decomposes the $\boldsymbol{X}$ matrix by a principal-component analysis (PCA), then fits an MLR model using the LVs instead of the raw data as predictors. PLS performs a simultaneous and interdependent PCA decomposition in both $\boldsymbol{X}$ (independent variables) and $\boldsymbol{Y}$ (dependent variables) matrices, in such a way that the information in the $\boldsymbol{Y}$-matrix is used for the optimal decomposition of the $\boldsymbol{X}$-matrix, and then performs the regression of $\boldsymbol{Y}$. Thus, in PLS two loading matrices and two score matrices are obtained; one is related to summarising the variation in the $\boldsymbol{X}$-space and the other in the $\boldsymbol{Y}$-space. They are called P-loading and $\boldsymbol{T}$-scores matrices in the $\boldsymbol{X}$-space and $\boldsymbol{Q}$-loading and $\boldsymbol{U}$-scores matrices in the $\boldsymbol{Y}$-space.

However, every PLS calibration results in a new set of $\boldsymbol{X}$ and $\boldsymbol{Y}$-loading, the $\boldsymbol{W}$ and $\boldsymbol{C}$ matrices in the $\boldsymbol{X}$ and $\boldsymbol{Y}$ space, respectively. The $\boldsymbol{W}$ matrix of weight vectors for $\boldsymbol{X}$ expresses how the information in each $X$-variable relates to the variation in the $\boldsymbol{Y}$ by the $\boldsymbol{U}$-scores, i.e. are the effective loading directly connected to building the relationship between $\boldsymbol{X}$ and $\boldsymbol{Y}$,

$$
w^{\prime}=\frac{u^{\prime} X}{u^{\prime} u}
$$

the superscript ' indicates the transposed vector.

The loading weights are specific to PLS, and are equal to the loadings in PCA and PCR. However, in PLS, the $\boldsymbol{W}$ loadings may or not be equal to the $\boldsymbol{P}$-loadings. This difference tells us how much the $\boldsymbol{Y}$-guidance has influenced the decomposition of X.

A crucial step in the application of PLS and PCR is the correct selection of the number of factors or LVs to retain in a model. The choice of a too-large number causes overfitting and bad predictions. On the contrary, a too-small number leads to underfitting and to the introduction of a systematic error. Because of this, two criteria were used and compared in order to select the optimal number of LVs. The first one chooses the number of LVs associated with the first local minimum predicted error sum of squares (PRESS) value (criterion I) for each model. The second one chooses the fewest number of LVs, such that the PRESS was not significantly greater than the PRESS from the model that yielded the minimum PRESS (criterion II). The $\boldsymbol{F}$-statistic was used to make the significance determination..$^{34}$

\section{Methods for data reduction}

As discussed previously, one of the most difficult tasks in variable selection is deciding which regions to include in the analysis. Sometimes knowledge about the chemistry of the sample can be used to intelligently choose a region optimum for analysis. However, in complex mixtures of components it can be extremely difficult to assign regions to specific components, because many of the signals will be overlapped. There are mathematical methods that can be used to select the best regions for calibration. Two of these methods are used here in combination with the PLS calibration model.

\section{Selection of regions based on the correlation plot for each component}

The principle of this selection is based on a calculation of the correlation between the chromatographic responses (absorbance) at every time, in the training chromatograms, with the concentrations of every component; i.e. the method generates a correlation chromatogram for each component of interest. As any time regression is performed, it is possible to calculate the determination coefficient $\left(r^{2}\right)$ which is a measure of how well the regression is correlated. This coefficient is defined by

$$
r^{2}=\left(\frac{\sum_{i=1}^{N}\left(\bar{y}_{i}-\bar{y}_{i}\right)^{2}}{\sum_{i=1}^{N}\left(y_{i}-\bar{y}_{i}\right)^{2}}\right)
$$

where $\hat{y}_{i}$ is the prediction of the concentration of interest in calibration sample $\mathrm{i}, y_{i}$ is the real concentration in calibration sample $\mathrm{i}$ and $\bar{y}_{i}$ is the mean of $Y$.

High-correlation ( $r^{2}$ close to 1$)$ values indicate chromatographic regions that are good for calibration of that component. Regions that show low or no correlation $\left(r^{2}\right.$ close to zero) should not be used for calibration, since they provide no information relevant to the components of interest. To optimise the regions, different levels of $r^{2}$ are selected and one keeps only 
regions with $r^{2}$ values exceeding those levels. After, the PLS model was applied previous mean centring of the data of the reduced regions. The model with the smallest PRESS value was selected as the optimum.

\section{Selection of regions with important loading weights}

In the classical PLS solution the two scores vectors, $\boldsymbol{t}$ and $\boldsymbol{u}$, are calculated to give the maximum covariance for each calculated LV. The key vector carrying the relation between $t$ and $\boldsymbol{u}$ is the weight vector $(\boldsymbol{w})$. The sizes of the elements in $\boldsymbol{w}$ are assumed to represent the importance of the corresponding variables in the model connecting the $\boldsymbol{X}$ and $\boldsymbol{Y}$-variables. A large $\boldsymbol{w}$-value is interpreted as a variable of high importance for that component. Similarly, small $\boldsymbol{w}$-values are thought to be of a little or no importance at all.

Our selection method is based on the calculations of the $\boldsymbol{w}$ loading weights corresponding to the models with the more significant number of LVs. The regions with large $\boldsymbol{w}$-values are the most important and are retained. To select the regions, the $\boldsymbol{w}$ loading weight plots, calculated for the different number of LVs, are superposed and several levels are chosen. The regions that exceed those levels are kept and the others are eliminated. After each selection, the PLS method was applied after centring the data. The model with the smallest prediction error value was selected.

\section{Experimental}

\section{Chemicals and solvents}

HPLC-grade solvents were used. The pesticide standards (pestanal quality) were obtained from Riedel-de Haën (Seelze, Germany). Solid standards were dissolved in acetonitrile (AcN) and stored at $4{ }^{\circ} \mathrm{C}$ in the dark, where they were stable for several months. HPLC-grade water was obtained in-house using a Milli-Q system (Millipore, Bedford, MA, USA). All solvents and samples were filtered through Millipore membrane filters before injection into the column.

Dichloromethane was of residue analysis quality (Riedel-de Haën). Phosphate buffer (0.01 M, pH 3.0) was prepared from anhydrous di-sodium hydrogenphosphate from Merck (Darmstadt, Germany) and ortho-phosphoric acid from Panreac (Barcelona, Spain) in Millipore Milli-Q purified water (HPLCgrade water); chemicals were of analytical-reagent grade or better.

\section{Instrumentation}

A Waters (Milford, MA, USA) Model 990 liquid chromatographic system, comprising a Model 600E constantflow pump, a Rheodyne six-port injection valve with a $20 \mu \mathrm{l}$ sample loop and a Model 990 photodiode array detector, were used. HPLC separations were carried out using a Microspher Chrompack $4.6 \times 100 \mathrm{~mm}(3 \mu \mathrm{m}$ particle size $) \mathrm{C}_{18}$ column. Data processing and instrument control were accomplished using the Waters Model 991 software. The spectral resolution used was $1.4 \mathrm{~nm}$ per diode in the range $200-350 \mathrm{~nm}$. The Grams/386 software package and PLSplus $\mathrm{V}^{2} .1 \mathrm{G}^{36}$ were used for the treatment of data.

A rotary vacuum evaporator (Buchi 461) with a thermostatic water-bath and a vacuum pump was also used.

\section{HPLC operating conditions}

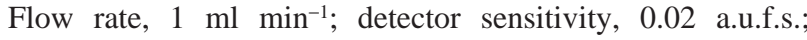
column at room temperature; wavelengths, $230 \mathrm{~nm}$; the mobile phase was $\mathrm{CH}_{3} \mathrm{CN} / 0.01 \mathrm{M}$ phosphate buffer ( $\left.\mathrm{pH} 3.0\right)(25 / 75$
Table 1 Concentration data $\left(\mu \mathrm{g} \mathrm{ml}^{-1}\right)$ of cyromazine $(\mathrm{C})$, oxamyl (O) and methomyl $(\mathrm{M})$ for the calibration and prediction set

\begin{tabular}{cccccccc}
\hline $\begin{array}{c}\text { Calibration } \\
\text { set }\end{array}$ & $\mathrm{C}$ & $\mathrm{O}$ & $\mathrm{M}$ & $\begin{array}{c}\text { Prediction } \\
\text { set }\end{array}$ & $\mathrm{C}$ & $\mathrm{O}$ & $\mathrm{M}$ \\
\hline $\mathrm{C} 1$ & 5 & 3 & 3 & $\mathrm{P} 1$ & 7 & 2 & 4 \\
C2 & 6 & 5 & 5 & $\mathrm{P} 2$ & 8 & 3 & 6 \\
C3 & 8 & 2 & 2 & $\mathrm{P} 3$ & 6 & 3 & 4 \\
C4 & 7 & 3 & 8 & $\mathrm{P} 4$ & 2.5 & 7 & 3.5 \\
C5 & 6 & 2 & 5 & $\mathrm{P} 5$ & 8 & 8 & 8 \\
C6 & 4 & 6 & 4 & $\mathrm{P} 6$ & 5.5 & 6 & 5.5 \\
C7 & 8 & 4 & 2 & - & - & - & - \\
C8 & 2 & 8 & 4 & - & - & - & - \\
C9 & 5 & 2 & 3 & - & - & - & - \\
C10 & 6 & 8 & 6 & - & - & - & - \\
C11 & 8 & 8 & 8 & - & - & - & - \\
C12 & 5 & 5 & 5 & - & - & - & - \\
C13 & 1 & 1 & 0 & - & - & - & - \\
C14 & 1 & 0 & 1 & - & - & - & - \\
C15 & 0 & 1 & 1 & - & - & - & - \\
\hline
\end{tabular}

$(\mathrm{v} / \mathrm{v}))$. The solvents were daily filtered through a $0.45 \mu \mathrm{m}$ cellulose acetate (Water) or politetrafluoroethylene (AcN) membrane filter before use, and degassed with helium both during and before use.

\section{Data}

A randomised design calibration matrix using a fifteen-sample set and a prediction set in the range $0-8 \mu \mathrm{g} \mathrm{ml}{ }^{-1}$ for cyromazine, oxamyl and methomyl was performed (Table 1). Volumes of $20 \mu \mathrm{l}$ were injected into the chromatographic system. The data were centred prior to the analysis by applying the PLS and PCR methods. The optimised PLS calibration models were used to determine the pesticides in the prediction set.

\section{Procedure for the determination of pesticides in groundwater}

A $500 \mathrm{ml}$ water sample $(\mathrm{pH} \mathrm{3})$ was extracted with three separate $100 \mathrm{ml}$ portions of dichloromethane by using a $1000 \mathrm{ml}$ separatory funnel and vigorous shaking for $2 \mathrm{~min}$. The extracts were combined and passed through a column of anhydrous sodium sulfate; then, the total extract was concentrated to dryness in a rotary vacuum evaporator. For HPLC analysis the residue was dissolved in $1 \mathrm{ml}$ of $\mathrm{AcN}$, and finally $20 \mu \mathrm{l}$ was injected.

\section{Results and Discussion}

\section{Chromatographic conditions}

A detailed study of the mobile-phase composition was carried out in order to perform the separation of five very polar pesticides. However, as discussed in the introduction, it is quite difficult to efficiently obtain their separation. Although we could separate two of them out of the rest, they were not resolved among themselves. This binary pesticide mixture ${ }^{37}$ was satisfactorily resolved by a cross-section approach. ${ }^{38}$

On the other hand, the three pesticides used in the study were easily eluted, and also showed overlapped signals with the initially tested mobile phases, aqueous $\mathrm{MeOH}$ or aqueous $\mathrm{AcN}$. In order to improve these results, $\mathrm{AcN}$ : water mobile phases, in which the $\mathrm{pH}$ of the aqueous portion was modified by using a

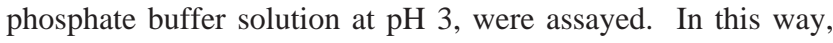
the three pesticides presented higher retention times, but remained overlapped in the time domain. In addition, sufficient 


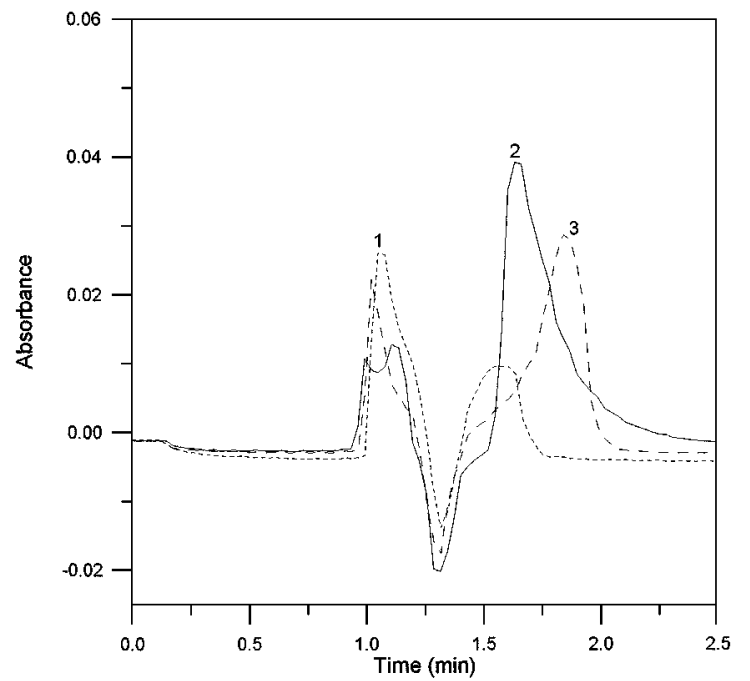

Fig. 1 LC chromatograms of $6 \mu \mathrm{g} \mathrm{ml} \mathrm{m}^{-1}$ of: (1) oxamyl; (2) cyromazine and (3) methomyl at $230 \mathrm{~nm}$.

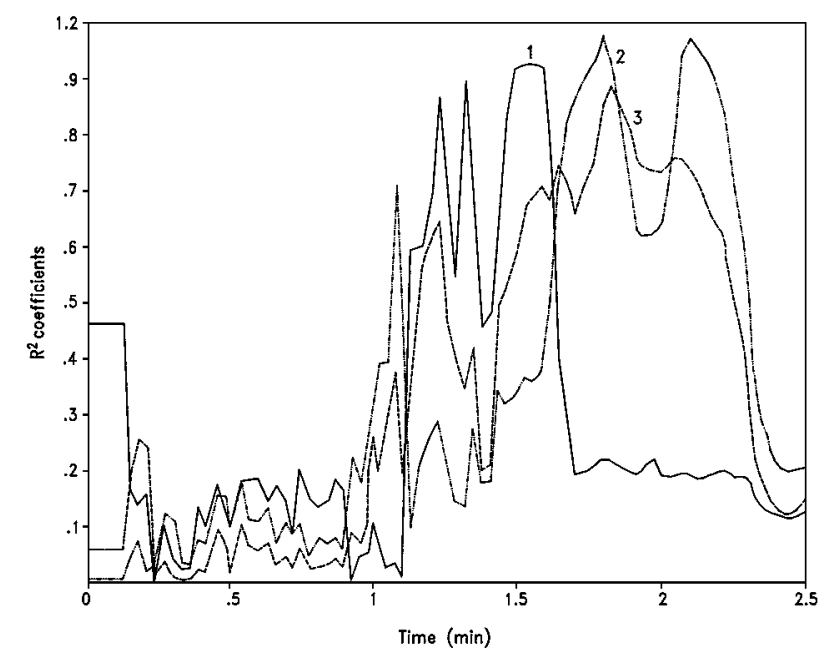

Fig. $2 r^{2}$ correlation chromatogram plots of: (1) oxamyl; (2) cyromazine and (3) methomyl.

selectivity between the analytes and solvent signals was not achieved.

Figure 1 shows LC chromatograms of cyromazine, oxamyl and methomyl obtained by injection of $20 \mu \mathrm{l}$ of pesticides standard solution with the mobile phase selected and at $230 \mathrm{~nm}$. Overlapping among the signals can be observed. This situation prevents applying the cross-section approach in order to obtain selective analytical information about each of the pesticides for resolving the mixture; we considered adequate to apply multivariate calibration methods (PLS and PCR) for the simultaneous determination of the three pesticides in the mixture.

\section{Full-spectrum multivariate calibration method}

PCR and PLS methods were applied, previous mean-centring of the data. The PRESS values obtained by cross-validation, and using two criteria for the selection of the optimal number of LVs, are summarised in Table 2. It can be seen that the best PRESS values were obtained with the PLS calibration method, which is in agreement with the findings by other workers. ${ }^{1,34,39}$
Table 2 PRESS and number of latent variables (in parentheses) in the calibration set, according the two criteria for selecting the optimum number of latent variables

\begin{tabular}{lcccc}
\hline Compound & \multicolumn{2}{c}{ Criterion I } & \multicolumn{2}{c}{ Criterion II } \\
\hline & PLS & PCR & PLS & PCR \\
Cyromazine & $4.37(3)$ & & $4.97(2)$ & \\
Oxamyl & $5.32(4)$ & $15.15(4)$ & $5.89(3)$ & $15.15(4)$ \\
Methomyl & $5.54(3)$ & & $6.06(2)$ & \\
\hline
\end{tabular}

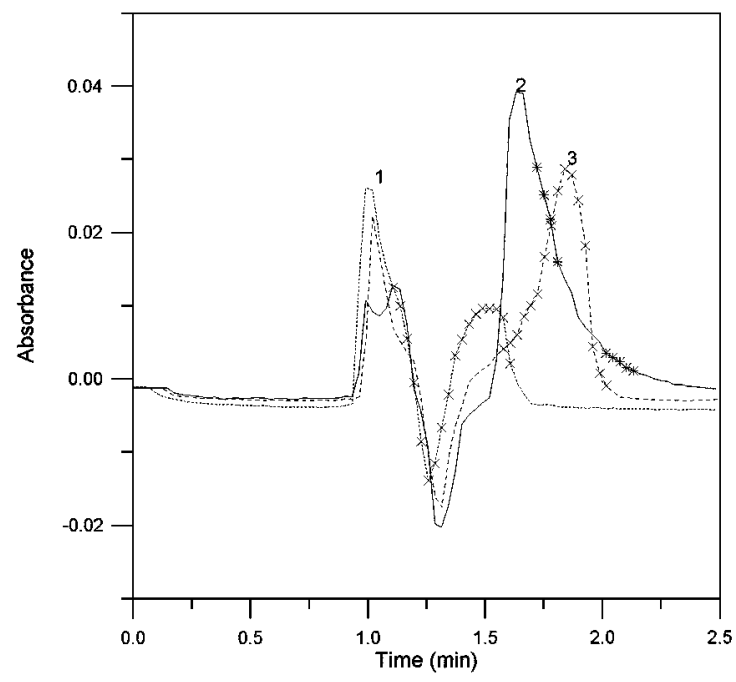

Fig. 3 Position of the selected variables on the pure chromatogram of: (1) cyromazine; (2) oxamyl and (3) methomyl according to the $r^{2}$ selection.

The optimal number of LVs found on the calibration set by PLS was 3 for methomyl and ciromazine and 4 for oxamyl if the rule of the first local minimum was applied (criterion I), and 2 for methomyl and ciromazine and 3 for oxamyl according to criterion II.

In order to determinate whether this increase in the number of LVs leads to overfitting, both PLS models were validated on independent samples of the prediction set. The results obtained by applying the PLS method and the two criteria for selecting the number of LVs, are summarised in Table 3. Non significant improvements of the predictions were observed when the number of LVs was higher (criterion I), although the PRESS values obtained according this criterion were smaller. Therefore, it can be dangerous to apply the rule of the first local minimum.

\section{Data-reduction methods}

Selection of regions based on the correlation plots. Plotted in Fig. 2 are the $r^{2}$ values for the pesticides as a function of the retention times. Clearly, there are time regions associated with large $r^{2}$ values for each analyte, and these should be chosen. In order to determine the optimum region for each one of the analytes, several PLS models were built using different numbers of variables. These were selected on the $r^{2}$ plot when they were larger than a preselected level of $r^{2}$.

Four different $r^{2}$ levels were chosen for ciromazine, oxamyl and methomyl. In the first step, it was decided to retain all variables, i.e. 87. The rest of the levels with their number of variables for each pesticide are given in Table 4. A PLS model, 
Table 3 Predictions (\%) of cyromazine, oxamyl and methomyl in the prediction set using the full PLS model and the reduced PLS models obtained with the $r^{2}(*)$ and loading weight $(* *)$ selection methods

\begin{tabular}{|c|c|c|c|c|c|c|c|c|c|c|c|c|}
\hline \multirow{4}{*}{$\begin{array}{c}\text { Prediction } \\
\text { set } \\
\text { P1 }\end{array}$} & \multicolumn{4}{|c|}{ Ciromazine } & \multicolumn{4}{|c|}{ Oxamyl } & \multicolumn{4}{|c|}{ Methomyl } \\
\hline & \multicolumn{2}{|c|}{ Full PLS } & \multirow{2}{*}{\multicolumn{2}{|c|}{$\begin{array}{ll}* & \\
& \text { (II) }\end{array}$}} & \multicolumn{2}{|c|}{ Full PLS } & \multirow{2}{*}{\multicolumn{2}{|c|}{$*{ }^{*}{ }^{* *}$}} & \multicolumn{2}{|c|}{ Full PLS } & \multirow{2}{*}{\multicolumn{2}{|c|}{ * $\quad$ (II) }} \\
\hline & (I) & (II) & & & (I) & (II) & & & (I) & (II) & & \\
\hline & 98.1 & 98.0 & 97.5 & 97.5 & 97.0 & 107.5 & 104.4 & 105.3 & 104.0 & 100.7 & 93.2 & 89.4 \\
\hline P2 & 106.0 & 105.0 & 95.2 & 96.0 & 111.7 & 100.7 & 102.2 & 103.2 & 103.3 & 102.2 & 93.8 & 90.8 \\
\hline P3 & 93.5 & 93.8 & 90.7 & 92.7 & 86.7 & 92.3 & 97.7 & 92.7 & 104.7 & 105.0 & 105.6 & 104. 1 \\
\hline $\mathrm{P} 4$ & 93.6 & 107.2 & 102.4 & 105.2 & 97.1 & 99.3 & 98.0 & 105.4 & 112.6 & 102.9 & 95.7 & 97.3 \\
\hline P5 & 101.7 & 103.9 & 99.2 & 98.2 & 101.7 & 103.9 & 104.3 & 98.7 & 104.9 & 102.5 & 102.8 & 106.2 \\
\hline P6 & 99.8 & 104.0 & 96.5 & 92.1 & 100.7 & 105.7 & 95.6 & 94.2 & 109.8 & 106.2 & 98.3 & 90.6 \\
\hline
\end{tabular}

I and II : criteria for the selection of the number of LVs.

Table 4 Number of variables (No. V.) number of latent variables (No. LVs) and PRESS values according to the criterion II to reduced PLS models obtained with the $r^{2}$ and loading weight selection

\begin{tabular}{|c|c|c|c|c|c|c|c|c|}
\hline \multicolumn{3}{|c|}{ Ciromazine } & \multicolumn{3}{|c|}{ Oxamyl } & \multicolumn{3}{|c|}{ Methomyl } \\
\hline No. V. & No. LVs & PRESS & No. V. & No. LVs & PRESS & No. V. & No. LVs & PRESS \\
\hline \multicolumn{9}{|c|}{$r^{2}$ selection } \\
\hline 87 & 2 & 4.97 & 87 & 3 & 5.89 & 87 & 2 & 6.06 \\
\hline 23 & 2 & 3.56 & 21 & 3 & 2.98 & 22 & 3 & 2.78 \\
\hline 9 & 3 & 1.84 & 15 & 4 & 5.92 & 16 & 3 & 1.91 \\
\hline 7 & 3 & 2.33 & 4 & 5 & 6.46 & 5 & 5 & 6.67 \\
\hline \multicolumn{9}{|c|}{ loading weight selection } \\
\hline 87 & 2 & 4.97 & 87 & 3 & 5.89 & 87 & 2 & 6.06 \\
\hline 13 & 3 & 3.67 & 33 & 3 & 4.98 & 33 & 2 & 5.57 \\
\hline 8 & 3 & 3.82 & 18 & 3 & 5.16 & 30 & 2 & 5.18 \\
\hline 4 & 2 & 4.65 & 5 & 2 & 12.1 & 10 & 5 & 7.4 \\
\hline
\end{tabular}

with mean-centring data, was made on each selected set and the prediction error values were calculated using criterion II for selecting the number of LVs. The model corresponding to the smallest prediction error is the optimum one, and therefore the variable region associated with this model is the optimum region to select. It can be observed that the prediction error improved down to the level with 9, 21 and 16 variables for ciromazine, oxamyl and methomyl, respectively; after that the prediction error values were increased.

To validate these reduced PLS models, they were applied to the resolution of the test set of synthetic ternary mixtures; the results obtained are summarised in Table 3. Satisfactory predictions were obtained with recovery values ranging from 90.7 to 102.4 for ciromazine, from 95.6 to 104.4 for oxamyl and from 93.2 to 105.6 for methomyl.

The retained times according this selection method are marked on the pure chromatogram in Fig. 3. It can check that the selected regions agree with the maximum height of the peaks of methomyl and oxamyl, although for this last pesticide some selected variables are located at representative regions of the solvent peak. However, the high chromatographic overlap in this system prevents one to find selective regions. Indeed, some variables retained do not agree with the maximum height of the peak compound (ciromazine).

Selection of regions based on the loading-weigh plots. In Fig. 4 the PLS loading weight vectors for ciromazine, oxamyl and methomyl calibrations, based on a different number of factors, are plotted as a function of the retention times. It can be seen that the greatest part of the information is obtained between 1 and 2 min.

As in the previous section, the goal was to retain those variable regions associated with large loading-weight values in the more important LVs. In order to determine how many variables are useful, several PLS models, built with different numbers of variables, were compared. These were included when they were larger than or equal to a particular level of loading-weight.

Four loading-weight levels were chosen for the three compounds (Table 4). It can be observed that the prediction error was reduced in the second loading-weight level selected for ciromazine and oxamyl, and in the third one for methomyl, i.e. 13, 33 and 30 variables, respectively. For those models with a smaller number of variables the prediction error was higher. This behavior was also observed with the above selection method; however, the prediction errors are now higher.

Table 3 presents the results of an analysis of the test set. The results indicate that a complete resolution of the mixture is also accomplished by the proposed reduced PLS models.

In Fig. 5 the selected times according this selection method of regions can be observed. These variables agree with the maximum height of the analyte peaks. However, for oxamyl and methomyl, some retained variables are situated at regions of the solvent signal.

Simultaneous determination of ciromazine, metomyl and oxamyl in groundwater

To check the reduced PLS models, in a real situation, they were applied to determine the three pesticides in environmental samples of groundwater. A trace enrichment step was necessary to obtain detection limits as low as ppb levels. To evaluate the potential of trace enrichment of the pesticides, samples of ultrapure water, spiked with $3.5 \mu \mathrm{g} 1^{-1}$ of the pesticides, were 

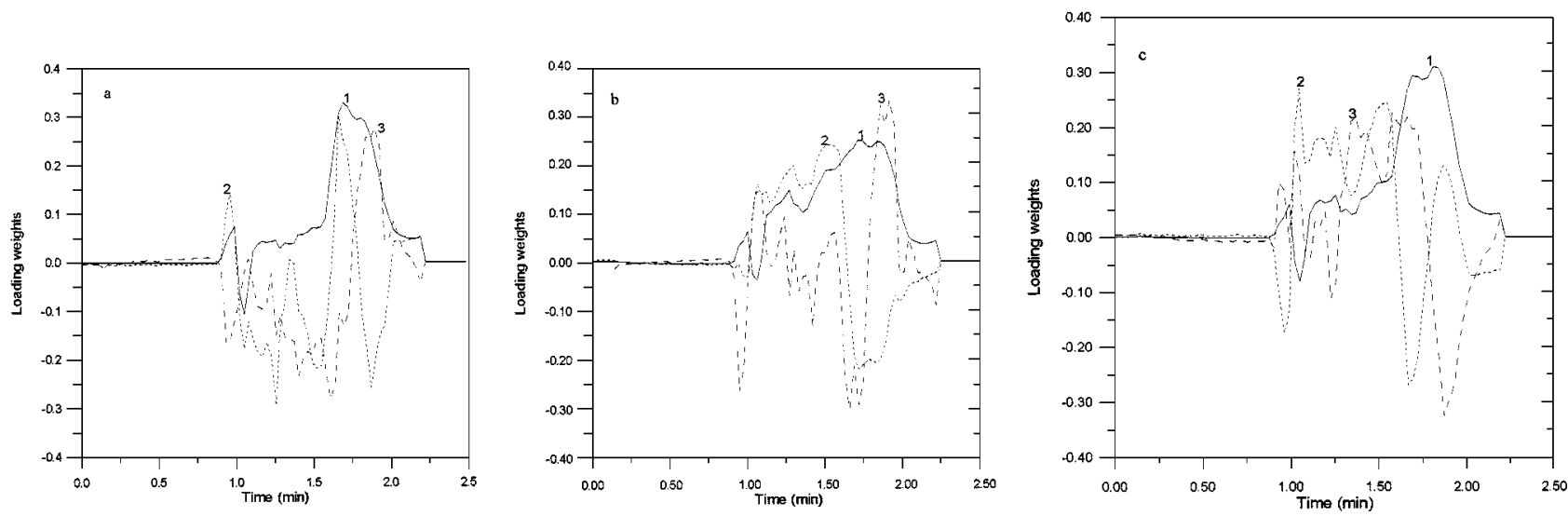

Fig. 4 Loading-weight plots for: (a) cyromazine; (b) oxamyl and (c) methomyl, obtained with a different number of latent variables $(1,2,3)$.

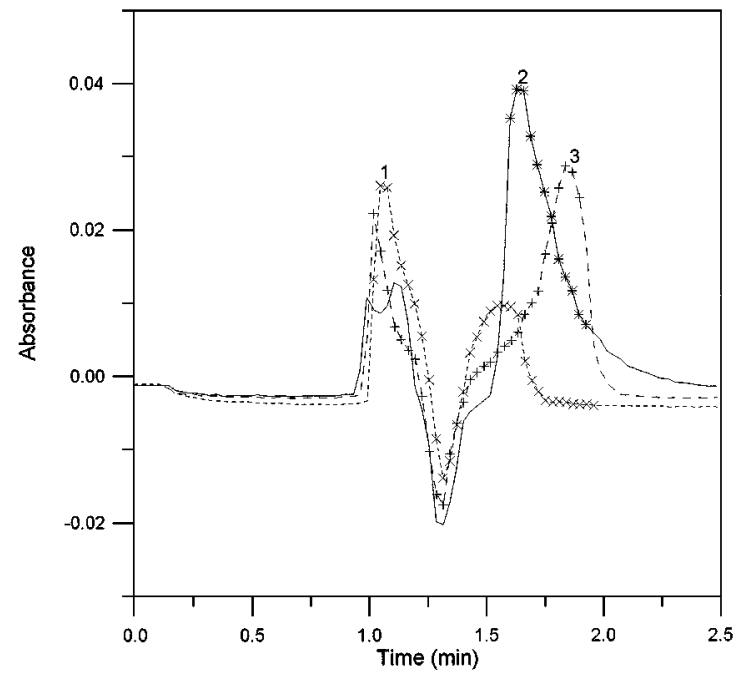

Fig. 5 Position of the selected variables on the pure chromatogram of: (1) cyromazine; (2) oxamyl and (3) methomyl according to the loading-weight selection.

analysed as described under Experimental section.

It was found (Table 5) that all of the compounds were effectively removed from their aqueous solutions using liquidliquid extraction (LLE), with recoveries ranging from 92.1 to $109.3 \%$.

The proposed method was applied to the determination of pesticide levels in groundwater samples of Almeria (Spain); the obtained chromatograms showed no peaks for the studied pesticides.

In short, the present research showed that the PLS method based on the whole original data matrix gives better prediction errors than does the PCR method. The use of the $F$-test for selecting the optimum number of LVs seems to be preferable to the first minimum PRESS, since it leads to simpler models with no loss in the quality of prediction. In addition, it is noteworthy that the PLS method performs better when the selection of regions is applied. Besides, of the two methods tested, selection based on the $r^{2}$ coefficient plots appears to be fundamentally better (better predictions). The selection methods described here have some advantages compared to other optimisation methods: (1) they permit one to select bands of variables instead of single variables, (2) they do not involve a time-consuming
Table 5 Recoveries in the preconcentration of $3.5\left(\mu \mathrm{g} 1^{-1}\right.$ of cyromazine, oxamyl and methomyl from groundwater

\begin{tabular}{rrrr}
\hline \multirow{2}{*}{ Method } & \multicolumn{3}{c}{ Recovery, \% } \\
\cline { 2 - 4 } & Cyromazine & Oxamyl & Methomyl \\
\hline Full-PLS & $95.4(6.6)$ & $104.8(5.3)$ & $109.3(6.5)$ \\
*PLS & $93.9(6.8)$ & $104.1(5.2)$ & $102.3(6.5)$ \\
**PLS & $92.1(6.9)$ & $102.9(5.6)$ & $105.1(6.2)$ \\
\hline
\end{tabular}

The results are averages of three determinations, with RSDs in parentheses. Reduced PLS models obtained with the $r^{2}$ selection (*) or loading weight selection $(* *)$.

optimisation method, such as genetic algorithm and (3) with these methods the selection of the best region can be studied separately for each component.

It is concluded that the PLS method is the most adequate to resolve this complex data set, where there is overlapping between analytes and the solvent peak at the same time, and that feature selection can be successfully applied in chromatographic analysis, not only in spectroscopic techniques, because the quality of PLS solutions is improved.

The method was applied to the determination of cyromazine, oxamyl and methomyl in groundwater samples with satisfactory results.

\section{Acknowledgement}

The authors are grateful to DGICYT (project PB95-1226) for the financial support.

\section{References}

1. E. V. Thomas and D. M. Haaland, Anal. Chem., 1990, 62, 1091.

2. P. MacLaurin, P. J. Worsfold, M. Crane, and P. Norman, Anal. Proc., 1992, 29, 65.

3. M. Martínez Galera, J. L. Martínez Vidal, A. Garrido Frenich, and P. Parrilla, Analyst[London], 1994, 119, 1189.

4. J. L. Martínez Vidal, M. D. Gil García, M. Martínez Galera, and A. Garrido Frenich, Anal. Lett., 1997, 30, 2409. 
5. D. T. Rossi and H. L. Pardue, Anal. Chim. Acta, 1985, 175, 153.

6. S. D. Frans and J. M. Harris, Anal. Chem., 1985, 57, 2680.

7. M. Otto and T. George, Anal. Chim. Acta, 1987, 200, 379.

8. P. J. Gemperline, J. Chemom., 1989, 3, 549.

9. J. H. Kalivas, N. Roberts, and J. M Sutter, Anal. Chem., 1989, 61, 2024

10. P. A. Salamin, H. Bartels, and P. Forster, Chemom. Intell. Lab. Syst., 1991, 11, 57.

11. C. B. Lucasius and G. Kateman, Tr. Anal. Chem., 1991, 10, 254.

12. R. Leardi, R. Boggia, and M. Terrile, J. Chemom., 1992, 6, 267.

13. P. J. Brown, J. Chemom., 1992, 7, 255.

14. M. Baroni, S. Clementi, G. Cruciani, G. Costantino, D. Riganelli, and E. Oberranch, J. Chemom., 1992, 6, 347.

15. M. Baroni, G. Costantino, G. Cruciani, D. Riganelli, R. Valigi, and S. Clementi, Quant. Struct.-Act. Relat., 1993 12,9 .

16. F. Lindgren, P. Geladi, S. Rännar, and S. Wold, J. Chemom., 1994, 8, 349.

17. C. B. Lucasius, M. L. M. Beckers, and G. Kateman, Anal. Chim. Acta, 1994, 286, 135.

18. R. Leardi, J. Chemom., 1994, 8, 65.

19. D. Jouan-Rimbaud, D. L. Massart, R. Leardi, and O. E. de Noord, Anal. Chem., 1995, 67, 4295.

20. U. Hörchner and J. H. Kalivas, Anal. Chim. Acta, 1995, 311,1 .

21. U. Hörchner and J. H. Kalivas, J. Chemom., 1995, 9, 283.

22. D. Jouan-Rimbaud, B. Walczak, D. L. Massart, I. R. Last, and K. A. Prebble, Anal. Chim. Acta, 1995, 304, 285.

23. A. Garrido Frenich, D. Jouan-Rimbaud, D. L. Massart, S. Kuttatharmmakul, M. Martínez Galera and J. L. Martínez Vidal, Analyst[London], 1995, 120, 2787.
24. L. Xu and Y. Schechter, Anal. Chem., 1996, 68, 2392.

25. V. Centner, D. L. Massart, O. E. de Noord, S. de Jong B. M. Vandeginste, and C. Sterna, Anal. Chem., 1996, 68, 3851.

26. A. S. Bangalore, R. E. Shaffer, G. W. Small, and M. A. Arnold, Anal. Chem., 1996, 68, 4200.

27. R. E. Shaffer and G. W. Small, Anal. Chem., 1997, 69, 236.

28. M. J. Arcos, M. C. Ortiz, B. Villahoz, and L. A. Sarabia, Anal. Chim. Acta, 1997, 339, 63.

29. J. M. Brenchley, U. Horchner, and J. H. Kalivas, Appl. Speectrosc., 1997, 51, 689.

30. D. H. Pote, T. C. Daniel, D. R. Edwards, J. D. Mattice, and D. B. Wickliff, J. Environ. Qual., 1994, 23, 101.

31. S. C. Stafford and W. Lin, J. Agric. Food Chem., 1992, 40, 1026.

32. M. Hiemstra and A. de Kok, J. Chromatogr., 1994, 667, 155.

33. A. de Kok and M. Hiemstra, J. AOAC Int., 1992, 75, 1063.

34. D. M. Haaland and E. V. Thomas, Anal. Chem., 1988, 60, 1993.

35. H. Martens and T. Naes, "Multivariate Calibration", 1991, Wiley, New York.

36. GRAMS-386 Software Package, Version 2.0, and Add-on Application PLSplus, V.2.1G, 1992, Galactic Industries, Salem.

37. M. Martínez Galera, A. Garrido Frenich, J. L. Martínez Vidal, and P. Parrilla Vázquez, J. Chromatogr., 1998, 799, 149.

38. A. Garrido Frenich, M. Martínez Galera, M. D. Gil García, J. L. Martínez Vidal, A. Muñoz de la Peña, and F. Salinas, Talanta, 1998, 46, 1329.

39. M. Martínez Galera, J. L. Martínez Vidal, A. Garrido Frenich, and M. D. Gil García, J. Chromatogr. A, 1997, $778,139$. 